\title{
Effects of Combination Treatment of Intravesical Resiniferatoxin Instillation and Hydrodistention in Patients with Refractory Painful Bladder Syndrome/Interstitial Cystitis: A Pilot Study
}

\author{
Byeong Kuk Ham, Jae Heon Kim, Mi Mi Oh, Jeong Gu Lee, Jae Hyun Bae \\ Department of Urology, Korea University College of Medicine, Seoul, Korea
}

\begin{abstract}
Purpose: Painful bladder syndrome/interstitial cystitis (PBS/IC) is a disabling disease of the urinary bladder, and its etiology and treatment are not yet established. Current medications used in the treatment of PBS/IC have shown limited efficacy. This prospective study investigated the efficacy of intravesical resiniferatoxin (RTX) in PBS/IC refractory to medical treatment.

Methods: Patients with proven PBS/IC refractory to traditional medical treatment were enrolled. By randomized trial, a total of 18 consecutive patients were divided into two groups: treatment with hydrodistention and intravesical RTX (group 1) or treatment with hydrodistension only (group 2). We assessed bladder pain by use of a visual analogue pain scale, the maximal urine flow rate, post-void residual urine volume, and a voiding diary before and 3 months after treatment.

Results: The median age of the 18 patients was $55.8 \pm 6.9$ years, and the median duration of symptoms before diagnosis was $3.6 \pm$ 1.6 years. Frequency, functional bladder capacity, and score on a 5-point pain scale were significantly improved at 3-month after treatment in both groups. Intravesical RTX instillation plus hydrodistention, compared with hydrodistention only, did not have a significant effect on the voiding symptoms or uroflowmetry of the patients but significantly improved scores on the pain scale. Conclusions: Intravesical RTX instillation plus hydrodistention was effective in relieving pain but was not effective in improving lower urinary tract symptoms. Further larger studies are needed to clarify the efficacy of combination treatment of intravesical RTX instillation and hydrodistention.
\end{abstract}

Keywords: Interstitial cystitis; Pain; Resiniferatoxin; Intravesical administration

\section{INTRODUCTION}

Interstitial cystitis (IC) is a chronic, disabling disease that is characterized by bladder pain, urgency, and frequency. IC was originally described by Hanash and Pool as a condition with severe urinary symptoms, reduced bladder capacity and typical cystoscopic findings of ulcer [1]. The diagnosis is made clinically and is confirmed by cystoscopy with hydrodistention and often by biopsy for exclusion of other pathologies. Controversies remain, however, over the definition, pathophysiology, and standard treatment of IC.
In 1987, the National Institute of Diabetes and Digestive and Kidney Diseases (NIDDK) proposed diagnostic criteria that are mainly based on exclusions [2], and that have been found to be too restrictive for clinical use. Recently, the European Society for the Study of Interstitial Cystitis proposed a new nomenclature and classification system [3]. Because pain is the fundamental feature of the condition, it was proposed that the name be changed to painful bladder syndrome (PBS).

Vanilloids modulate the activity of sensory neurones. Resiniferatoxin (RTX) is an ultra-potent analogue of the chilli pepper extract capsaicin, that causes less pain on instillation and is there-
Corresponding author: Jae Hyun Bae

Department of Urology, Korea University Ansan Hospital, Korea University

College of Medicine, 123 Jeokgeum-ro, Danwon-gu, Ansan 425-707, Korea

Tel: +82-31-412-5190 / Fax: +82-31-412-5194

E-mail: urobae@genetherapy.or.kr
This is an Open Access article distributed under the terms of the Creative Commons Attribution Non-Commercial License (http://creativecommons.org/licenses/by-nc/3.0/) which permits unrestricted non-commercial use, distribution, and reproduction in any medium, provided the original work is properly cited. 
fore easier to use. However, the treatment outcome of RTX remains contradictory.

Refractory PBS/IC is defined as persistent, unacceptable symptoms despite oral or intravesical therapy. According to the 2010 IC guidelines [4], more aggressive modalities such as hydrodistention, neuromodulation, and intravesical botulinum toxin are recommended for refractory PBS/IC. Although hydrodistention, which is a common treatment, is included as an interventional treatment, there are limitations in its scientific basis [5].

The primary aim of this study was to investigate the safety and effectiveness of the combination therapy of intravesical RTX instillation and hydrodistention in patients with refractory PBS/ IC. To our knowledge, this is the first randomized trial of intravesical RTX instillation and hydrodistention.

\section{MATERIALS AND METHODS}

This study was part of a prospective, longitudinal, observational study. Eighteen women who met the NIDDK consensus research criteria for IC and were refractory to classic medical treatment were studied. By randomized trial, all patients were divided into two groups: treatment with hydrodistention and intravesical RTX (group 1, $\mathrm{n}=8$ ) or treatment with hydrodistension only (group 2, $\mathrm{n}=10$ ). Informed consent was obtained from each subject, who were advised of the potential adverse effects of hydrodistention and intravesical RTX instillation, and the study was approved by the Institutional Review Board. Before treatment, a voiding diary, uroflowmetry parameters, and visual analogue pain scale (VAS) scores were recorded. VAS scores and a 3-day voiding diary were recorded at 1 month and 3 months after treatment. The endpoint of this study was the recurrence of pain that required treatment, including medical or interventional treatment.

The combination treatment of RTX intravesical instillation and hydrodistention was done under general anesthesia. The method of hydrodistention as follows. The bladder was filled with solution $(300 \mathrm{~mL} /$ pack of 3\% D-sorbitol solution; Baxter, Deerfield, IL, USA) to $80 \mathrm{~cm} \mathrm{H}_{2} \mathrm{O}$. Drop speed was approximately $30 \mathrm{~mL} / \mathrm{min}$. During the procedure, the cystoscope was fixed at the back or side walls. The bladder was filled until it began to leak from the urethra around the cystoscope. At that time, the liquid was let out. The cystoscope was fixed at the same position and dropping was stopped. The liquid was discharged slowly from the bladder at $30 \mathrm{~mL} / \mathrm{min}$ and and the procedure was carefully repeated. The expected goal of bladder capacity was $400 \mathrm{~mL}$ or $500 \mathrm{~mL}$. If the functional bladder capacity (FBC) was $<200 \mathrm{~mL}$, the expected goal for bladder capacity was 400 $\mathrm{mL}$, and if $\mathrm{FBC}$ was more than $200 \mathrm{~mL}$, the expected goal for bladder capacity was $500 \mathrm{~mL}$.

All RTX doses were administrated as a 10\% ethanolic solution. Fifty milliliters of the drug solution was administrated after complete urine drainage. RTX was administrated through a silicon urinary catheter and was held in the bladder for $30 \mathrm{~min}$ utes. Then the bladder was drained and irrigated with normal saline. A new urethral Foley catheter was indwelled overnight.

The vital signs of the patients were checked regularly during the procedures. The urethral Foley catheter was removed on the second day after the operation, and the patient was discharged on the third day.

The primary outcome measures assessed were preoperative and postoperative average frequency, average bladder volume from a 3-day voiding diary, and VAS score (patients were asked to grade the severity of pain on a linear scale from 0 to 5 with 0 meaning no pain and 5 meaning the worst pain). Therapeutic outcome was judged to be positive when pain was relieved at 3 months of follow-up and no aggravation or new occurrence of bladder pain was noted through 3 months of follow-up. Recurrence was judged to be a condition in need of treatment such as medication or interventional treatment to control bladder pain.

Statistical analysis was performed by using SPSS ver. 18.0 (SPSS Inc, Chicago, IL, USA). Data are reported as mean values with standard deviations, because normality of the distribution of continuous variables was proven by the Kolmogorov-Smirnov test. Comparisons between preoperative and postoperative data were performed by use of the Wilcoxon signed-rank test and the Pearson chi-square test. To identify the variables affecting recurrence and pain aggravation, the Mann-Whitney $\mathrm{U}$ test was conducted.

\section{RESULTS}

The median age of the 18 patients was $55.8 \pm 6.9$ years and the mean duration of symptoms before diagnosis was $3.6 \pm 1.6$ years. There were no clinically meaningful differences among the two groups in the severity of IC symptoms. All patients had undergone previous therapy to control their symptoms without a beneficial effect. The characteristics of the patients in each group before treatment are described in Table 1 .

No voiding parameters showed improvement at 3 months of 
Table 1. Characteristics of the patients in the two groups

\begin{tabular}{lccc}
\hline Characteristic & $\begin{array}{c}\text { Group 1 } \\
(\mathrm{n}=8)\end{array}$ & $\begin{array}{c}\text { Group 2 } \\
(\mathrm{n}=10)\end{array}$ & P-value \\
\hline Age (yr) & $54.5 \pm 9.1$ & $56.9 \pm 7.8$ & 0.81 \\
Duration of IC symptoms (yr) & $3.5 \pm 1.5$ & $3.8 \pm 1.3$ & 0.77 \\
Duration of previous Tx (yr) & $2.8 \pm 1.6$ & $3.0 \pm 1.8$ & 0.64 \\
Frequency (/day) & $16.8 \pm 6.7$ & $15.6 \pm 7.6$ & 0.76 \\
Nocturia (/night) & $4.4 \pm 1.7$ & $4.4 \pm 2.5$ & 0.72 \\
Pain score & $3.3 \pm 0.8$ & $3.5 \pm 1.1$ & 0.67 \\
FBC (mL) & $210.4 \pm 34.7$ & $239.0 \pm 36.0$ & 0.21 \\
Qmax (mL/sec) & $14.8 \pm 5.7$ & $16.3 \pm 6.7$ & 0.71 \\
PVR (mL) & $15.0 \pm 19.4$ & $14.2 \pm 13.8$ & 0.97
\end{tabular}

Values are presented as mean \pm standard deviation.

IC, interstitial cystitis; Tx, treatment; FBC, functional bladder capacity; Qmax, maximal urine flow rate; PVR, post-void residual urine volume.

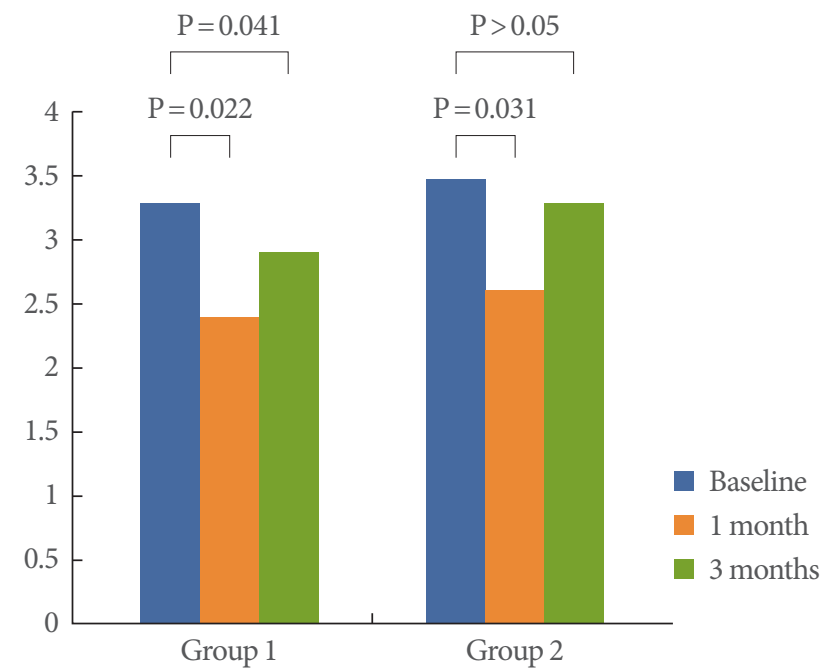

Fig. 1. Differences in pain scores between baseline and 1 month and 3 months of follow-up.

follow-up in either group, but scores on the 5-point pain scale showed improvement at both 1 month and 3 months follow-up in group 1 (Table 2, Fig. 1). There were no significant differences in nocturia, maximal urine flow rate, or post-void residual urine volume (Table 2).

Frequency showed improvement at 1 month in both groups ( $P=0.02,0.03$, respectively), but did not show improvement at 3 months (Fig. 2). FBC showed improvement at 1 month in both groups $(P=0.02,0.03$, respectively), but did not show improvement at 3 months (Fig. 3). Bladder pain was significantly relieved at both 1 month and 3 months of follow-up in group 1 ( $\mathrm{P}=0.022,0.041$, respectively), but was relieved only at 1 month
Table 2. Changes from before treatment to the 3 months followup after treatment in the two groups

\begin{tabular}{|c|c|c|c|}
\hline Parameter & Group $1(n=8)$ & Group $2(n=10)$ & P-value ${ }^{a}$ \\
\hline \multicolumn{4}{|c|}{ NO. of frequency (/day) } \\
\hline Baseline & $16.8 \pm 6.7$ & $15.6 \pm 7.6$ & \multirow{4}{*}{$>0.05$} \\
\hline After 3 mo & $15.4 \pm 2.9$ & $14.8 \pm 6.0$ & \\
\hline Delta & $-0.4 \pm 4.1$ & $-6.6 \pm 2.5$ & \\
\hline P-value ${ }^{\mathrm{b})}$ & $>0.05$ & $>0.05$ & \\
\hline \multicolumn{4}{|c|}{ No. of nocturia (/night) } \\
\hline Baseline & $4.4 \pm 1.7$ & $4.4 \pm 2.5$ & \multirow{4}{*}{$>0.05$} \\
\hline After 3 mo & $3.6 \pm 1.5$ & $3.1 \pm 1.4$ & \\
\hline Delta & $-0.8 \pm 0.7$ & $-1.3 \pm 1.4$ & \\
\hline P-value ${ }^{b)}$ & $>0.05$ & $>0.05$ & \\
\hline \multicolumn{4}{|l|}{ Pain score } \\
\hline Baseline & $3.3 \pm 0.8$ & $3.5 \pm 1.1$ & \multirow{4}{*}{0.024} \\
\hline After 3 mo & $2.9 \pm 0.4$ & $3.3 \pm 0.7$ & \\
\hline Delta & $-0.5 \pm 0.8$ & $-0.2 \pm 0.8$ & \\
\hline P-value ${ }^{\text {b) }}$ & 0.041 & $>0.05$ & \\
\hline \multicolumn{4}{|l|}{$\mathrm{FBC}(\mathrm{mL})$} \\
\hline Baseline & $210.4 \pm 34.7$ & $239.0 \pm 36.0$ & \multirow{4}{*}{$>0.05$} \\
\hline After 3 mo & $222.9 \pm 36.9$ & $242.0 \pm 50.2$ & \\
\hline Delta & $5.5 \pm 15.8$ & $3.0 \pm 17.6$ & \\
\hline P-value ${ }^{\text {b) }}$ & $>0.05$ & $>0.05$ & \\
\hline \multicolumn{4}{|c|}{$\operatorname{Qmax}(\mathrm{mL} / \mathrm{sec})$} \\
\hline Baseline & $14.8 \pm 5.7$ & $16.3 \pm 6.7$ & \multirow{4}{*}{$>0.05$} \\
\hline After 3 mo & $15.2 \pm 5.4$ & $16.2 \pm 5.4$ & \\
\hline Delta & $0.3 \pm 1.0$ & $-0.1 \pm 2.3$ & \\
\hline P-value ${ }^{b)}$ & $>0.05$ & $>0.05$ & \\
\hline \multicolumn{4}{|l|}{ PVR (mL) } \\
\hline Baseline & $15.0 \pm 19.4$ & $14.2 \pm 13.8$ & \multirow{4}{*}{$>0.05$} \\
\hline After 3 mo & $13.8 \pm 11.8$ & $13.2 \pm 9.6$ & \\
\hline Delta & $-1.2 \pm 17.6$ & $-1.0 \pm 4.2$ & \\
\hline P-value $e^{\text {b) }}$ & $>0.05$ & $>0.05$ & \\
\hline
\end{tabular}

Values are presented as mean \pm standard deviation.

FBC, functional bladder capacity; Qmax, maximal urine flow rate; PVR, post-void residual urine volume.

${ }^{\text {a) }} \mathrm{P}$-value by Mann-Whitney U test. ${ }^{\text {b) }} \mathrm{P}$-value was analyzed by Wilcoxon signed rank test.

of follow-up in group $2(\mathrm{P}=0.031)$ (Fig. 1).

No serious adverse effects (bladder rupture, sepsis) occurred after the treatments, but hematuria (2 patients in group 1) and bladder pain (1 patient in group 2 ) occurred. These side effects disappeared within 2 days. 


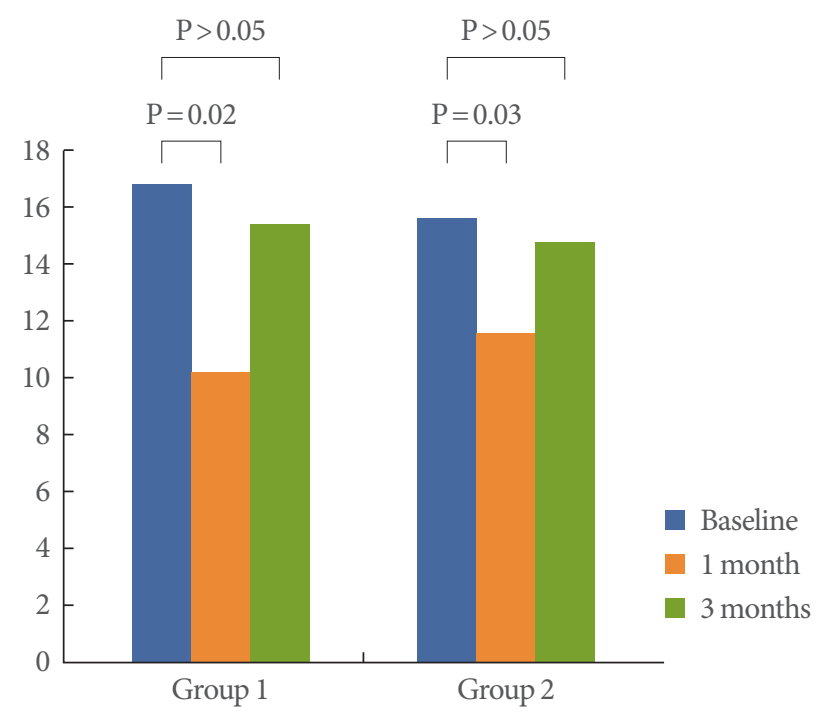

Fig. 2. Differences in frequency numbers between baseline and 1 month and 3 months of follow-up.

\section{DISCUSSION}

$\mathrm{PBS} / \mathrm{IC}$ is a chronic inflammatory disease that causes symptoms of pain in the bladder or pelvis with or without urgency, frequency, and nocturia [6]. The prevalence in women was estimated at around 18.1 cases per 100,000 females in a Finnish survey. The prevalence in both sexes is 10.6 cases per 100,000 persons with the annual incidence of new cases being 1.2 cases per 100,000 persons. Severe cases account for about $10 \%$ of the total and most studies show a female to male preponderance of 5:1 or greater [7]. PBS impairs quality of life, causes sleep dysfunction, depression, and creates anxiety, sexual and social problems [8]. Treatment of PBS can be classified into conservative, oral medical therapy, intravesical drug instillation, neuromodulation and surgical therapy. Despite the many treatment strategies, there are no general standard treatments yet. Many drugs are used empirically, and only a few of them have been studied in randomized controlled trials.

Refractory PBS/IC is defined as persistent, unacceptable symptoms despite oral or intravesical therapy. According to the 2010 IC guideline [4], more aggressive treatment modalities such as interventional treatment are recommended.

Hydrodistention of the bladder has been used for many years, not only for diagnosis but also for treatment of PBS [9], but recent studies have reported poor results, with only a small number of patients reporting symptom improvement for only a short period of time $[5,10]$. Moreover hydrodistention has limitations

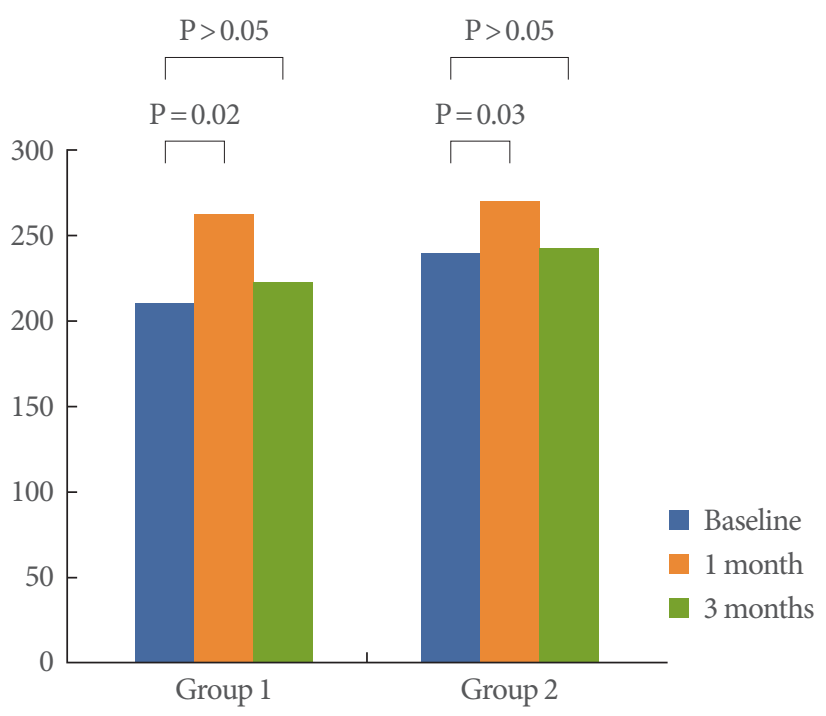

Fig. 3. Differences in functional bladder capacity $(\mathrm{mL})$ between baseline and 1 month and 3 months of follow-up.

in its scientific basis and could yield diverse adverse events including both mild events (e.g., hematuria, bladder pain) and serious events (e.g., bladder rupture, sepsis). According to the 2011 American Urological Association guideline, high-pressure, longduration hydrodistention should not be offered (grade $\mathrm{C}$ recommendation) [11]. In our study, patients underwent hydrodistention under direct observation by cystoscope after general anesthesia. To reduce adverse events by hydrodistention, the expected goal of bladder capacity was determined by consulting the patient's individual voiding diary. If FBC was under 200 $\mathrm{mL}$, the expected goal for bladder capacity was $400 \mathrm{~mL}$, and if FBC was more than $200 \mathrm{~mL}$, the expected goal for bladder capacity was $500 \mathrm{~mL}$.

RTX is an ultra-potent analogue of the chilli pepper extract capsaicin, that causes less pain on instillation and is therefore easier to use. Vanilloids modulate the activity of sensory neurons and pain-mediating C-fibers [12]. Studies have been conducted on the vanilloid receptor-1, which is activated by vanilloid agonists, such as capsaicin or its analogue, RTX. The efficacy of RTX has been shown in small clinical trials [13,14], but different results were reported from two randomized, doubleblind, placebo-controlled studies conducted with instillation of a single dose of RTX in different concentrations [15,16]. In those two studies, both study groups were followed up for 3 months with evaluation several times. No significant improvement in symptoms was found in either of the trials, but both reported dose-dependent increases in instillation pain. Thus, until now, 
the treatment outcome of RTX has been various and contradictory.

Intravesical RTX instillation in idiopathic detrusor overactivity has recently been studied $[17,18]$. Considering the shared pathogenesis of the mediation of C-fibers between idiopathic detrusor overactivity and PBS/IC, these studies remind us of RTX treatment. Silva et al. [17] in their open-label study reported the efficacy of a single $50 \mathrm{nM}$ intravesical instillation of RTX, with improvement in clinical and urodynamic parameters. Rios et al. [18] in their double-blind, randomized, placebocontrolled trial, studied $50 \mathrm{nM}$ intravesical RTX and reported no differences in efficacy compared with placebo. One proven fact concerning intravesical RTX treatment is that its shortterm outcome is favorable. This is why Peng and Kuo [19] studied the efficacy of multiple intravesical low-doses of RTX, which yield favorable efficacy in relieving lower urinary tract symptoms in refractory PBS/IC.

In our study, intravesical RTX with hydroditention significantly improved voiding symptoms and bladder pain in patients with refractory PBS/IC at 1 month of follow-up. However, no favorable outcomes on voiding symptoms were seen at 3 months. Bladder pain was relieved at 3 months, but longer follow-up is crucial for the evaluation of clinical efficacy. Our study was a pilot study and the first trial of combination therapy of intravesical RTX instillation and hydrodistention.

Our study did have some limitations. First, we could not determine the effect of intravesical RTX instillation only compared with RTX plus hydrodistetion. Considering the dose of RTX, the effect of RTX could be small. Because of this being a pilot study, we could not examine a higher dose together with hydrodistention. Second, the number of patients was small. Third, although we designed a randomized pilot study, we did not use placebo instillation. However, the commonly used placebo material, ethanol, affects bladder capacity and micturition pressure by itself. Also, considering the anesthesia state, the placebo effect could be minimal. Last, urodynamic study was not performed of sensory and motor function of the bladder. Larger studies are needed, including higher-dose RTX treatment or repeated low-dose RTX in refractory PBS/IC.

In conclusion, intravesical RTX instillation plus hydrodistention was effective in relieving pain over hydrodistention only. Although the shorter term outcome of both treatments showed improvements in clinical efficacy, only the combination treatment improved pain. Further larger studies are needed or other noninvasive combination methods must be devised to increase clinical efficacy in refractory PBS/IC.

\section{CONFLICT OF INTEREST}

No potential conflict of interest relevant to this article was reported.

\section{REFERENCES}

1. Hanash KA, Pool TL. Interstitial cystitis in men. J Urol 1969;102: 427-8.

2. Gillenwater JY, Wein AJ. Summary of the National Institute of Arthritis, Diabetes, Digestive and Kidney Diseases Workshop on Interstitial Cystitis, National Institutes of Health, Bethesda, Maryland, August 28-29, 1987. J Urol 1988;140:203-6.

3. van de Merwe JP, Nordling J, Bouchelouche P, Bouchelouche K, Cervigni M, Daha LK, et al. Diagnostic criteria, classification, and nomenclature for painful bladder syndrome/interstitial cystitis: an ESSIC proposal. Eur Urol 2008;53:60-7.

4. Hanno P, Lin A, Nordling J, Nyberg L, van Ophoven A, Ueda T, et al. Bladder Pain Syndrome Committee of the International Consultation on Incontinence. Neurourol Urodyn 2010;29:191-8.

5. Cole EE, Scarpero HM, Dmochowski RR. Are patient symptoms predictive of the diagnostic and/or therapeutic value of hydrodistention? Neurourol Urodyn 2005;24:638-42.

6. Theoharides TC. Treatment approaches for painful bladder syndrome/interstitial cystitis. Drugs 2007;67:215-35.

7. Kusek JW, Nyberg LM. The epidemiology of interstitial cystitis: is it time to expand our definition? Urology 2001;57(6 Suppl 1):95-9.

8. Nickel JC, Tripp DA, Pontari M, Moldwin R, Mayer R, Carr LK, et al. Psychosocial phenotyping in women with interstitial cystitis/ painful bladder syndrome: a case control study. J Urol 2010;183: 167-72.

9. Franksson C. Interstitial cystitis: a clinical study of fifty-nine cases. Acta Chir Scand 1957;113:51-62.

10. Ottem DP, Teichman JM. What is the value of cystoscopy with hydrodistension for interstitial cystitis? Urology 2005;66:494-9.

11. Hanno PM, Burks DA, Clemens JQ, Dmochowski RR, Erickson D, Fitzgerald MP, et al. AUA guideline for the diagnosis and treatment of interstitial cystitis/bladder pain syndrome. J Urol 2011;185:216270.

12. Lazzeri M, Beneforti P, Benaim G, Maggi CA, Lecci A, Turini D. Intravesical capsaicin for treatment of severe bladder pain: a randomized placebo controlled study. J Urol 1996;156:947-52.

13. Apostolidis A, Gonzales GE, Fowler CJ. Effect of intravesical Resin- 
iferatoxin (RTX) on lower urinary tract symptoms, urodynamic parameters, and quality of life of patients with urodynamic increased bladder sensation. Eur Urol 2006;50:1299-305.

14. Lazzeri M, Beneforti P, Spinelli M, Zanollo A, Barbagli G, Turini D. Intravesical resiniferatoxin for the treatment of hypersensitive disorder: a randomized placebo controlled study. J Urol 2000;164(3 Pt 1):676-9.

15. Chen TY, Corcos J, Camel M, Ponsot Y, Tu le M. Prospective, randomized, double-blind study of safety and tolerability of intravesical resiniferatoxin (RTX) in interstitial cystitis (IC). Int Urogynecol J Pelvic Floor Dysfunct 2005;16:293-7.

16. Payne CK, Mosbaugh PG, Forrest JB, Evans RJ, Whitmore KE, Antoci JP, et al. Intravesical resiniferatoxin for the treatment of interstitial cystitis: a randomized, double-blind, placebo controlled trial.
J Urol 2005;173:1590-4.

17. Silva C, Ribeiro MJ, Cruz F. The effect of intravesical resiniferatoxin in patients with idiopathic detrusor instability suggests that involuntary detrusor contractions are triggered by C-fiber input. J Urol 2002;168:575-9.

18. Rios LA, Panhoca R, Mattos D Jr, Srugi M, Bruschini H. Intravesical resiniferatoxin for the treatment of women with idiopathic detrusor overactivity and urgency incontinence: a single dose, 4 weeks, double-blind, randomized, placebo controlled trial. Neurourol Urodyn 2007;26:773-8.

19. Peng CH, Kuo HC. Multiple intravesical instillations of low-dose resiniferatoxin in the treatment of refractory interstitial cystitis. Urol Int 2007;78:78-81. 